\title{
Advances in Chemotherapy against Advanced or Metastatic Colorectal Cancer
}

\author{
Kenji Omura \\ Department of General and Cardiothoracic Surgery, Kanazawa University School of Medicine, Kanazawa, Japan
}

\author{
Key Words \\ Colorectal cancer · Chemotherapy · 5-Fluorouracil • \\ Leucovorin • Oxaliplatin • Irinotecan • Molecular targeting
}

\begin{abstract}
In the early 1990s, some prospective controlled trials revealed the superiority of chemotherapy for survival compared with best supportive care for advanced or metastatic colorectal carcinoma. Until recently, 5-fluorouracil (5-FU) and leucovorin (LV) were the standard therapies against advanced or metastatic colorectal cancer. Theoretically, LV should increase the antitumor activity of 5-FU, although this effect of LV addition has been controversial. A meta-analysis which analyzed 21 randomized controlled trials revealed that a combination of 5-FU and LV doubled the response rate compared with 5-FU alone (from 11 to 21\%) and prolonged the median survival time by about 1 month (from 10.5 to 11.7 months). Chemotherapy against advanced or metastatic colorectal cancer has steadily advanced after the introduction of triplet regimens containing 7-ethyl-10-[4-(1-piperidino)-1-piperidino]carbonyloxy-camptothecin (CPT-11) and (trans-R,R-1,2-diamine cyclohexane)oxalatoplatinum(II) (LOHP). For LV/5-FU/CPT-11, the regimen in which 5-FU is administered with continuous infusion (FOLFIRI) is preferred compared with the 5-FU bolus infusion. According to the results of the randomized controlled trial comparing FOLFIRI followed by FOLFOX6 and the reverse sequence, FOLFOX6 followed by FOFIRI, in the treatment of advanced colorectal cancer, FOLFIRI and FOLFOX are now considered to have al-
\end{abstract}

most the same efficacy in the treatment of advanced or metastatic colorectal cancer. FOLFIRI followed by FOLFOX or FOLFOX followed by FOLFIRI provide a median survival time of about 21 months in advanced or metastatic colorectal cancer. Both an anti-vascular endothelial growth factor monoclonal antibody, bevacizumab, and an anti- epidermal growth factor receptor monoclonal antibody, cetuximab, should prolong the survival of advanced or metastatic colorectal cancer by 2-3 months in combination with FOLFIRI or FOLFOX. However, from the viewpoint of medical economics, because of the high acquisition costs in relation to clinical benefits, antibodies are unlikely to represent a costutility solution. New agents, including macromolecule agents, small-molecule agents and vaccines, will be introduced alongside chemotherapy against colorectal cancer. Subsequently, clinical researchers will have to consider the cost-utility of these agents. Copyright $\odot 2008$ S. Karger AG, Basel

\section{Introduction}

Colorectal cancer is the third leading cause of cancer death in Japan. Approximately $40 \%$ of all patients develop metastatic disease. Consequently, chemotherapy which provides obvious prolongation of survival in metastatic colorectal cancer is quite useful. Until recently, 5FU, which has been available for over 40 years, and folinate calcium [leucovorin (LV)] have been the standard therapy. However, LV/5-FU showed no major impact on

\section{KARGER}

Fax +4161306 1234

E-Mail karger@karger.ch

www.karger.com (c) $2008 \mathrm{~S}$. Karger AG, Basel

0012-2823/08/0775-0013\$24.50/0

Accessible online at:

www.karger.com/dig
Kenji Omura

Department of General and Cardiothoracic Surgery

Kanazawa University School of Medicine, 13-1 Takaramachi

Kanazawa, 920-8641 (Japan)

Fax +81 76222 6833, E-Mail omura@med.kanazawa-u.ac.jp 
survival. Chemotherapy against advanced or metastatic colorectal cancer has steadily improved with the introduction of triplet regimens containing irinotecan [7-ethyl-10-(4-[1-piperidino]-1-piperidino)carbonyloxy-camptothecin (CPT-11)] or oxaliplatin [(trans-R,R-1,2-diamine cyclohexane)oxalatoplatinum(II) (L-OHP)]. Chemotherapy for advanced or metastatic colorectal cancer has been supplemented with other promising agents: monoclonal antibodies against vascular endothelial growth factor (VEGF) and epidermal growth factor receptor (EGFR). This review describes progress in chemotherapy against advanced or metastatic colorectal cancer over the last 2 decades.

\section{Rationale of Chemotherapy against Advanced or Metastatic Colorectal Cancer}

Until the 1980s, the effect of chemotherapy on advanced or metastatic colorectal cancer was unclear. Some prospective controlled trials were performed to estimate the survival benefit of chemotherapy in patients with advanced, recurrent or metastatic colorectal cancer. Scheithauer et al. [1] reported the results of a prospective randomized trial which compared the survival and quality of life in patients given combination chemotherapy and best supportive care (BSC) and in patients given only BSC. The chemotherapy consisted of 5-FU, folinic acid and cisplatin. Forty previously untreated patients with locally recurrent or metastatic colorectal cancer were enrolled. The patients were randomized to receive chemotherapy and BSC or only BSC at a ratio of 2:1. Chemotherapy consisted of 5-FU, LV and cisplatin. Overall survival (OS) was significantly longer for patients given chemotherapy and BSC (11.0 months) than for those receiving only BSC ( 5.0 months, $\mathrm{p}=0.006)$. Mild to moderate gastrointestinal toxicity was commonly observed in the chemotherapy group. However, there was no significant difference in the quality of life scores between the 2 groups. According to the results of this study, chemotherapy against advanced or metastatic colorectal cancer was considered to prolong survival without reducing quality of life.

\section{5-FU and LV}

$\mathrm{LV}$ is a reduced folate which is easily metabolized to methylenetetrahydrofolate $\left(\mathrm{CH}_{2} \mathrm{H}_{4} \mathrm{PteGlu}\right) . \mathrm{CH}_{2} \mathrm{H}_{4}$ PteGlu is an essential cofactor in the inhibition of thymidylate synthase (TS, EC 2.1.1.45) by 5-fluoro-2'-deoxyuridine$5^{\prime}$-monophosphate (FdUMP), which is an active form of 5 -FU [2]. TS catalyzes the reductive methylation of deoxyuridine monophosphate to form deoxythymidine monophosphate, which is the rate-limiting reaction in de novo synthesis of pyrimidine nucleotides. $\mathrm{CH}_{2} \mathrm{H}_{4}$ PteGlu is a cofactor in this reaction to provide the 1-carbon unit, $\mathrm{CH}_{2}$. FdUMP binds to the active site of TS with higher affinity than dUMP, which is the native substrate of TS [3]. However, FdUMP is not methylated due to binding of fluoride to the 5th position of uracil, where hydrogen is substituted by the 1-carbon unit $\mathrm{CH}_{3}$ with the catalysis of TS. Then, FdUMP and $\mathrm{CH}_{2} \mathrm{H}_{4} \mathrm{PteGlu}$ bind tightly and occupy the active site of TS. Thus, an inactive ternary complex is formed with TS, FdUMP and $\mathrm{CH}_{2} \mathrm{H}_{4}$ PteGlu, and TS is competitively inhibited. As the amount of reduced folates, including $\mathrm{CH}_{2} \mathrm{H}_{4} \mathrm{PteGlu}$, is limited, TS inhibition by FdUMP is insufficient due to lack of $\mathrm{CH}_{2} \mathrm{H}_{4} \mathrm{PteGlu}$. Other reduced folates such as $\mathrm{CH}_{3} \mathrm{H}_{4}$ PteGlu, $\mathrm{CHH}_{4}$ PteGlu and $\mathrm{CHOH}_{4}$ PteGlu play essential roles to transfer the 1-carbon unit in important reactions to maintain homeostasis and to synthesize nucleotides. Consequently, simultaneous use of reduced folate and LV should increase the antitumor activity of 5-FU.

Many studies revealed that the combination of LV and 5 -FU (LV/5-FU) increased the response rate (RR) of advanced, recurrent or metastatic colorectal cancer compared with 5-FU alone. However, the effect of LV addition is controversial because the RR of LV/5-FU were 20$30 \%$, and the chemotherapy barely affected the median survival time (MST). A meta-analysis which analyzed 21 randomized controlled trials (RCTs) revealed that the combination of 5-FU and LV increased the RR 2-fold compared with 5-FU alone (from 11 to $21 \%$ ) and prolonged the MST by 1 month (from 10.5 to 11.7 months) [4].

Several regimens have been developed for LV/5-FU. The differences between these regimens are mainly the method of administration of 5-FU, bolus or infusion. Madajewicz et al. [5] reported weekly administration of LV/5-FU with bolus 5-FU. Their regimen is now called the Roswell Park Memorial Institute regimen. In the USA, another LV/5-FU regimen, in which LV and 5-FU are administered for 5 consecutive days for 4 weeks, was also developed [6]. The latter regimen is called the Mayo regimen. The Roswell Park Memorial Institute and Mayo regimens are comparable in terms of clinical effect, but the Mayo regimen is considered more toxic. On the other hand, LV/5-FU regimens, in which continuous infusion 
of 5-FU was applied, were developed in Europe. De Gramont et al. [7] reported a unique regimen for LV/5-FU, in which LV $200 \mathrm{mg} / \mathrm{m}^{2}$ was administered with a 2-hour infusion followed by a 5 -FU $400 \mathrm{mg} / \mathrm{m}^{2}$ bolus and 22 -hour continuous $5-\mathrm{FU} 600 \mathrm{mg} / \mathrm{m}^{2}$ infusion, repeated on days 1 and day 2 for 2 weeks. They named this regimen LV5FU2. Recently, a simplified LV5FU2 regimen (sLV5FU2) has been introduced. LV infusion and bolus 5 -FU are performed only on day 1 , followed by 46 -hour continuous infusion of 5-FU in sLV5FU2.

Another LV/5-FU regimen, in which 5-FU is administered with continuous infusion, was introduced by the Association of Medical Oncology of the German Cancer Society (AIO) [8]. In the AIO regimen, $\mathrm{LV} 500 \mathrm{mg} / \mathrm{m}^{2}$ was administered with 2 -hour infusion followed by 24 -hour continuous infusion of 5 -FU $2,600 \mathrm{mg} / \mathrm{m}^{2}$ weekly for 6 weeks during 8 weeks. It was unclear which was better for prolongation of survival, bolus 5-FU or infusional 5-FU, in LV/5-FU against advanced or metastatic colorectal cancer.

The clinical effect of chemotherapy against colorectal cancer has made rapid progress strides with the development of the combination regimens, LV/5-FU/CPT-11 and LV/5-FU/L-OHP, described below. In these triplet regimens, especially for LV/5-FU/CPT-11, infusional 5-FU demonstrated a better outcome than bolus 5-FU [9].

\section{CPT-11 and LV/5-FU/CPT-11 against Recurrent or Metastatic Colorectal Cancer}

Camptothecin is a plant alkaloid-derived antitumor agent. CPT was purified from extraction of Camptotheca acuminata. CPT-11 is a water-soluble analogue of CPT, which showed a higher antitumor activity and less toxicity than CPT [10]. Topoisomerase I, which is the target enzyme of CPT-11, regulates the superhelical density of DNA by transiently nicking 1 strand of the DNA helix. Topoisomerase I may be involved in many aspects of DNA metabolism, including transcription, replication and recombination. Consequently, inhibition of topoisomerase I causes DNA breakage and sequential cell death [11]. Although CPT-11 alone has considerable antitumor activity against colorectal cancer, it showed a marked combination effect with LV/5-FU.

Saltz et al. [12] reported the results of a comparison of LV/5-FU/CPT-11, LV/5-FU and CPT-11 alone as first-line chemotherapy for metastatic colorectal cancer. A total of 683 patients were randomized to the LV/5-FU/CPT-11 group (LV $20 \mathrm{mg} / \mathrm{m}^{2}$ bolus, 5 -FU $500 \mathrm{mg} / \mathrm{m}^{2}$ bolus and
CPT-11 $125 \mathrm{mg} / \mathrm{m}^{2}$ infusion weekly for 4 weeks during 6 weeks; $\mathrm{n}=231$ ), the LV/5-FU group (LV $20 \mathrm{mg} / \mathrm{m}^{2}$ bolus, 5 -FU $425 \mathrm{mg} / \mathrm{m}^{2}$ bolus daily for 5 consecutive days for 4 weeks; $\mathrm{n}=226$ ) and the CPT-11 group (CPT-11 $125 \mathrm{mg} /$ $\mathrm{m}^{2}$ infusion weekly for 4 weeks during 6 weeks; $\mathrm{n}=226$ ). As compared with the LV/5-FU group, the LV/5-FU/CPT11 group showed a significantly higher RR (39 vs. 21\%; $\mathrm{p}<0.001)$ and a longer MST (14.8 vs. 12.6 months; $\mathrm{p}=$ 0.04). The results for the CPT-11 group were similar to those for the LV/5-FU group. The schedule of LV/5-FU/ CPT-11 used in this study is called the Saltz regimen or IFL (fig. 1). Although the efficacy of IFL was sensational at that time, this regimen was too toxic. The mortality rate within 60 days from the start of IFL was $6.7 \%$.

Infusional 5-FU and LV combined with CPT-11 is called FOLFIRI. The first report of FOLFIRI was sLV5FU2 with CPT-11 (fig. 1) [13]. FOLFIRI was introduced as a third-line chemotherapy for metastatic colorectal cancer. Among 33 patients who underwent FOLFIRI, 2 had partial responses and the RR was $6 \%$. Twenty patients (61\%) had stable disease and the disease control rate was $67 \%$. From the start of FOLFIRI, the median progression-free survival (PFS) was 18 weeks and the MST was 43 weeks. National Cancer Institute Common Toxicity Criteria (NCI-CTC) toxicities grade 3-4 were nausea (15\%), diarrhea (12\%) and neutropenia (15\%). Overall, 10 patients (30\%) experienced grade 3-4 toxicity.

FOLFIRI is now recognized as one of the first-line chemotherapy regimens for recurrent or metastatic colorectal cancer, it has almost the same efficacy as LV/5-FU/LOHP described in detail below.

\section{L-OHP and LV/5-FU/L-OHP against Recurrent or Metastatic Colorectal Cancer}

L-OHP has potential against many cancer cell lines and tumors, including some that are primarily resistant to cisplatin and carboplatin [14]. The 1,2-diaminocyclohexane $(\mathrm{DACH})$ ligand provides higher lipophilicity compared to cis- and carboplatin, which is expressed by a large volume of distribution and a slower excretion through the kidneys. The lipophilic properties of oxaliplatin can also contribute to differences in general toxicity, as well as to an altered cellular uptake [15].

The DACH ligand plays a crucial role in the cytotoxic profile of oxaliplatin, forming intra- or interstrand adducts in the ultimate target DNA. The adducts are recognized and processed differently from those of cisplatin and carboplatin. Furthermore, due to the methylene 
Fig. 1. Drug administration schemata of IFL (a) and FOLFIRI (b). ci = Continuous infusion.

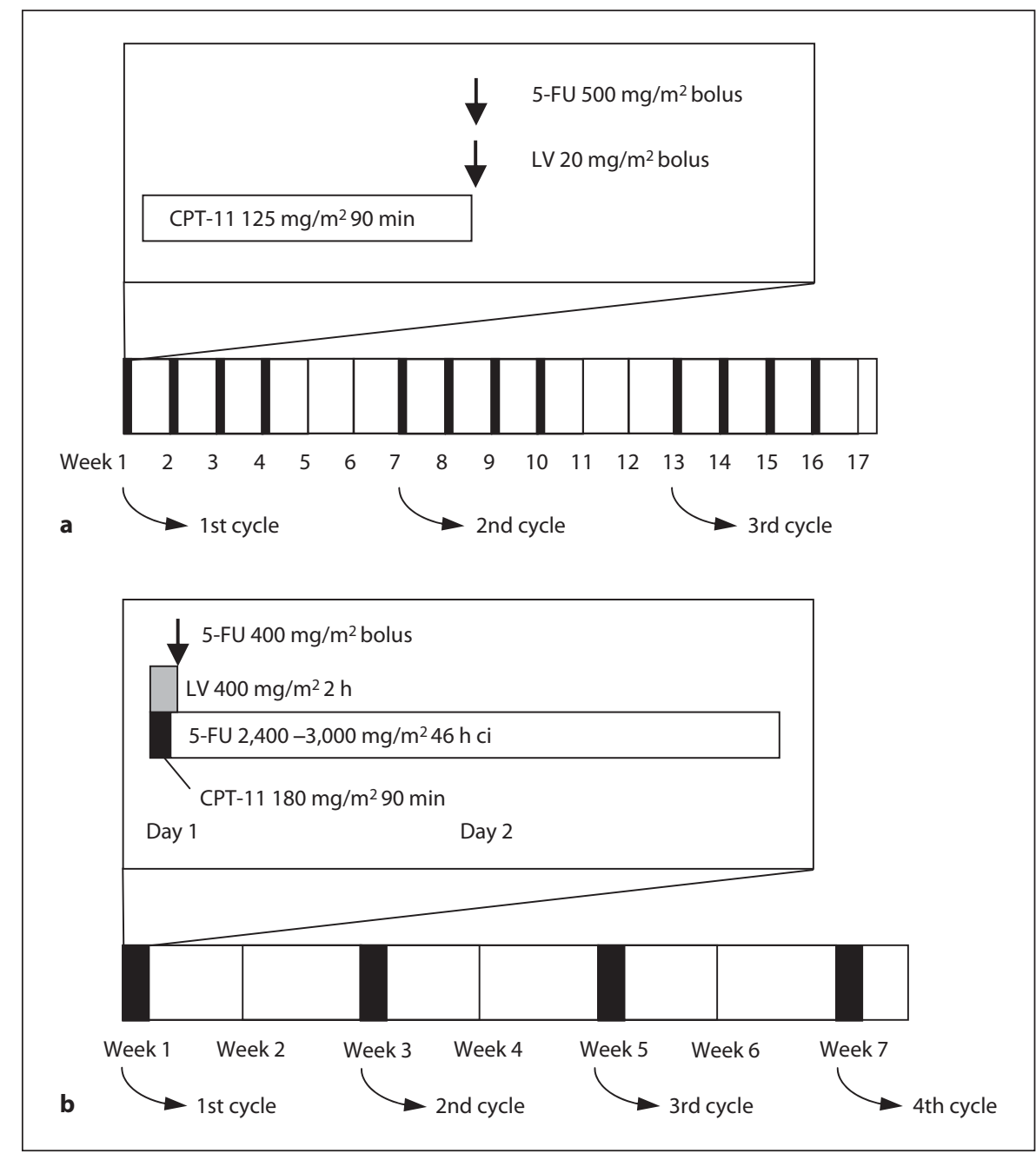

units of the cyclohexane ring, an unpolar region is formed at the DNA. Both features contribute to the different recognition and repair of adducts formed between oxaliplatin and the DNA [15].

The platinum complexes with the amino groups in the trans position display better cytotoxic and anticancer activities than the cis- $(R, S)$ isomer. Furthermore, the trans$(R, R)$ isomer shows significantly more potent antitumor activity than the trans-S,S congener. The DACH ligand provides such a marked influence on the pharmacological profile and effectiveness of oxaliplatin in primarily cis- and carboplatin-resistant tumors that derivatization at the trans- $R, R$-cyclohexane-1,2-diamine moiety could lead to improved anticancer properties [15]. L-OHP alone showed an insufficient clinical effect on recurrent or metastatic colorectal cancer. However, it is widely known that $\mathrm{L}-\mathrm{OHP}$ has a synergistic effect when it is used with 5-FU/
LV. L-OHP has unique adverse effects, including sensory neuropathy, lack of coordination due to pheripheral nerve disturbances and allergy.

De Gramont et al. [16] first reported the efficacy of LV/5-FU/L-OHP and named their regimen FOLFOX. Originally, FOLFOX consisted of LV $200 \mathrm{mg} / \mathrm{m}^{2}$ 2-hour infusion, followed by 5 -FU $300 \mathrm{mg} / \mathrm{m}^{2}$ bolus and $300 \mathrm{mg} /$ $\mathrm{m}^{2} 22$-hour infusion both on days 1 and 2 for 2 weeks. Then, in the absence of toxicity, 5-FU was increased to a $400-\mathrm{mg} / \mathrm{m}^{2}$ bolus and 22 -hour infusion in course 2 and to $500 \mathrm{mg} / \mathrm{m}^{2}$ in course 3 and from course 4 maintained at $500 \mathrm{mg} / \mathrm{m}^{2}$. In the first phase II study, 37 patients with advanced measurable colorectal cancer received the original FOLFOX. One patient $(2.7 \%)$ had a complete response, 19 (51.4\%) had partial responses, and the RR was $54 \%$. The MST was 18 months and $21 \%$ of the patients were alive at 2 years. After that, several FOLFOX regi- 
Fig. 2. Drug administration schemata. a FOLFOX4. b FOLFOX6 (the dose of L$\mathrm{OHP}$ is $85 \mathrm{mg} / \mathrm{m}^{2}$ in modified FOLFOX6). c FOLFOX7.

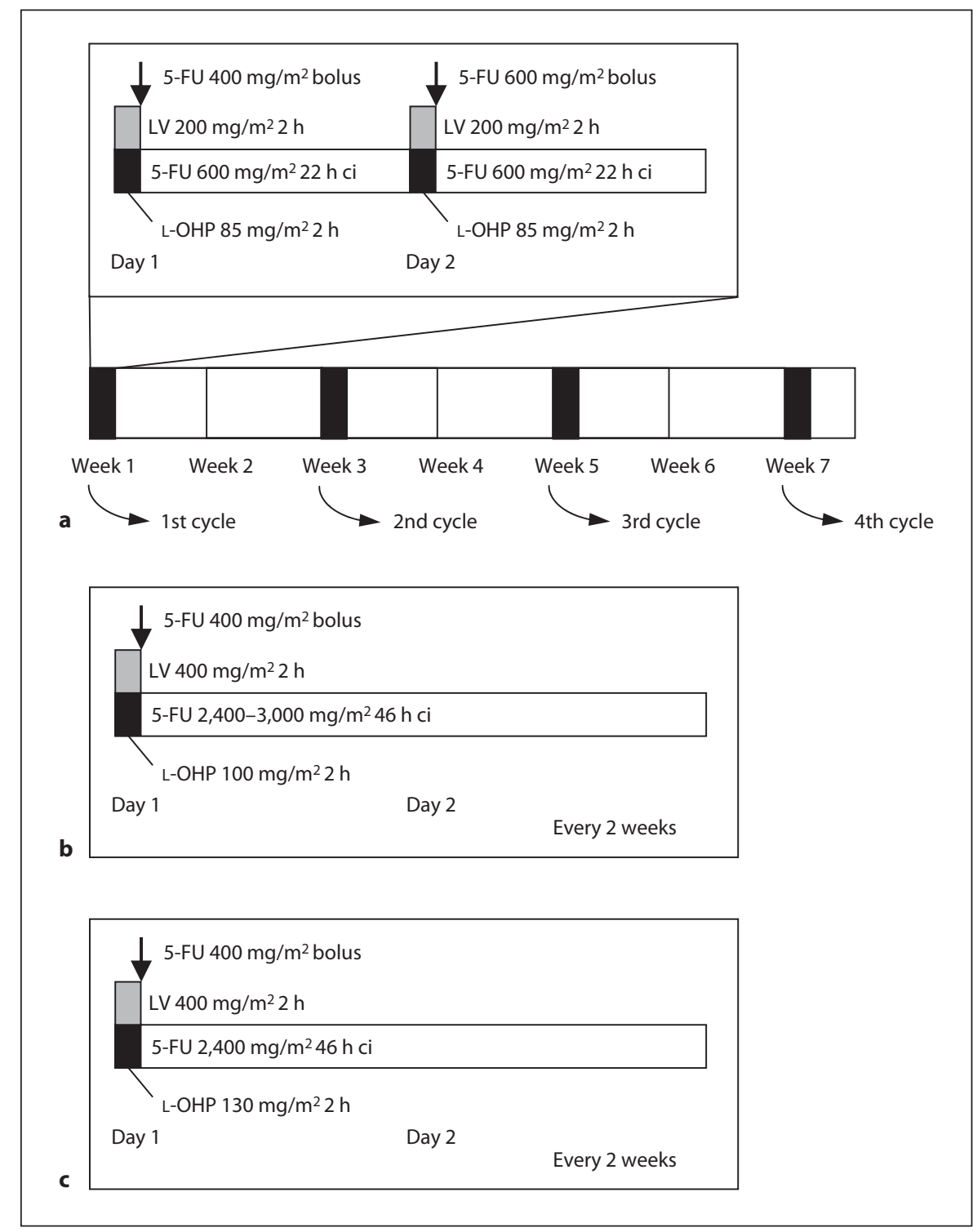

mens were developed (fig. 2). FOLFOX4, FOLFOX6, mFOLFOX6 and FOLFOX7 are now used for the treatment of recurrent or metastatic colorectal cancer. mFOLFOX6 is a regimen in which the dose of L-OHP is 85 $\mathrm{mg} / \mathrm{m}^{2}$, instead of $130 \mathrm{mg} / \mathrm{m}^{2}$ in FOLFOX6.

FOLFOX4 is the most common regimen applied to many RCTsl. In the first report, of the 57 patients who received FOLFOX4, there were 51 eligible patients and the RR was $23.5 \%(12 / 51)$ [17]. The major toxicities with FOLFOX4 were peripheral neuropathy and neutropenia. The incidence of grade 3-4 neutropenia was $36.9 \%$. The median PFS was 5.1 months and the median OS was 11.1 months.

Advances in Chemotherapy against Colorectal Cancer
De Gramont et al. [18] reported the results of an RCT which compared LV5FU2 and FOLFOX as first-line chemotherapy for advanced colorectal cancer. A total of 420 patients were enrolled in the LV5FU2 group $(n=210)$ and FOLFOX4 group $(\mathrm{n}=210)$. Patients allocated to FOLFOX4 had a significantly longer PFS (median, 9.0 vs. 6.2 months, $\mathrm{p}=0.0003$ ) and a better RR (50.7 vs. $22.3 \%, \mathrm{p}=$ 0.0001 ) when compared with the control LV5FU2. However, the improvement in OS was not significant (median, 16.2 vs. 14.7 months, $\mathrm{p}=0.12$ ).

The clinical effects of FOLFOX regimens have been compared with other regimens, and they are now recognized as one of the first-line chemotherapy regimens for 
recurrent or metastatic colorectal cancer. However, it has been pointed out that patients who cannot be reintroduced to the second cycle due to the adverse effect of LOHP had a bad prognosis. The occurrence of sensory neuropathy is significant because it may cause patients who are continuing to respond to treatment to discontinue treatment. Thus, new regimens, OPTIMOX1 [19] and OPTIMOX2 [19], have been developed to increase the rate of reintroduction without decreasing the clinical effect.

OPTIMOX1 was developed to avoid the problem of neurotoxicity induced by L-OHP so as to eliminate the main factor which disturbs the reintroduction of a regimen containing L-OHP. OPTIMOX1 (arm B) consisted of 6 cycles of dose-intense FOLFOX7 ( 1 cycle $=2$ weeks) followed by 12 cycles of maintenance (LV/5-FU2; 1 cycle $=2$ weeks) and subsequent reintroduction of FOLFOX7 for another 6 cycles. OPTIMOX1 was compared with FOLFOX4 ( $\operatorname{arm~A}$ ) in previously untreated metastatic colorectal cancer administered until progression or occurrence of unacceptable toxicity [19]. A total of 620 patients were enrolled in this study and randomized to receive $\operatorname{arm} A(n=311)$ and $\operatorname{arm} B(n=309)$. The median PFS were 9.0 and 8.7 months, and the MST were 19.3 and 21.2 months in arm A and arm B, respectively (no significance). The RR were $58.5 \%$ in arm $A$ and $59.2 \%$ in arm B (no significance). NCI-CTC grade 3 sensory neuropathy was observed in $17.9 \%$ of the patients in arm B and in $13.3 \%$ of those in arm $B(p=0.12)$. For the patients who received arm B, oxaliplatin was reintroduced in only $40.1 \%$ of the patients but achieved partial response or stable disease in $69.4 \%$ of them. According to these results, they concluded that L-OHP could be safely stopped after 6 cycles in a FOLFOX regimen.

In 2007, at the American Society of Clinical Oncology (ASCO) annual meeting, results comparing OPTIMOX1 and OPTIMOX2 in the chemotherapy against metastatic colorectal cancer were reported [20]. OPTIMOX2 was designed to introduce a complete cessation of chemotherapy after FOLFOX. OPTIMOX2 consisted of 6 cycles of FOLFOX7 (total 12 weeks) followed by complete cessation of chemotherapy (chemotherapy-free interval) and reintroduction of FOLFOX7 before the tumor progression reached the baseline. A total of 202 patients were enrolled in this study and randomized to the OPTIMOX1 group $(n=99)$ and OPTIMOX2 group $(n=103)$. The median duration of the chemotherapy-free interval in the OPTIMOX2 group was 4.6 months. The RR of OPTIMOX1 and OPTIMOX2 were 63 and 61\%, respectively. However, both median PFS and MST of the patients who received OPTIMOX1 were significantly longer than in those who received OPTIMOX2. The chemotherapy-free interval was not recommended from these results. Further study is needed to fully evaluate stop and go administration of L-OHP.

\section{LV/5-FU/CPT-11 versus LV/5-FU/L-OHP}

Goldberg et al. [9] reported the results of an RCT comparing the effects of LV/5-FU/CPT-11, LV/5-FU/L-OHP and CPT-11/L-OHP against metastatic colorectal cancer (N9741). A total of 795 patients were enrolled in this study. IFL was employed as LV/5-FU/CPT-11 $(\mathrm{n}=264)$, FOLFOX4 as LV/5-FU/L-OHP ( $\mathrm{n}=267)$, and irinotecan $200 \mathrm{mg} / \mathrm{m}^{2}$ and oxaliplatin $85 \mathrm{mg} / \mathrm{m}^{2}$ every 3 weeks (IROX) as CPT-11/L-OHP ( $\mathrm{n}=264)$. The RR of the patients receiving FOLFOX4 (45\%) was significantly higher than for those receiving IFL $(31 \%, \mathrm{p}=0.002)$ or IROX $(35 \%, p=0.03)$. The $\mathrm{RR}$ of patients receiving IFL and IROX did not differ $(\mathrm{p}=0.34)$. The time to progression (TTP) was significantly longer for the patients receiving FOLFOX4 (median, 8.7 months) than for those receiving IFL (median, 6.7 months; $\mathrm{p}=0.0014$ ). The median TTP for the patients receiving IROX was almost the same as for those receiving IFL (median, 6.5 months, $\mathrm{p}>0.50$ ), and the TTP for the patients receiving FOLFOX4 was significantly longer than for those receiving IROX $(\mathrm{p}=$ 0.001). The MST for the patients receiving IFL (15.0 months) was significantly shorter than for those receiving FOLFOX4 (19.5 months, $\mathrm{p}=0.0001)$ and those receiving IROX (17.4, $\mathrm{p}=0.04$ for comparison with IFL). On the other hand, the MST for the patients receiving FOLFOX4 did not differ from the MST of those receiving IROX $(p=0.09)$. The patients who received IFL had significantly higher rates of diarrhea, vomiting, nausea and febrile neutropenia and significantly lower rates of paresthesias and neutropenia compared with the patients treated with FOLFOX4. The rates of grade 3 or higher toxicity for the patients receiving IROX were similar to those for the patients receiving IFL. The second-line treatments were not specified by the protocol in this study. A high proportion $(60 \%)$ of the patients treated with FOLFOX4 received CPT-11 as the second-line therapy, and fewer patients (24\%) receiving IFL were treated with L-OHP regimens as the second-line therapy. Regardless, the results of N9741 showed the superiority of FOLFOX4 compared with IFL and IROX in the treatment of metastatic colorectal cancer.
18

Digestion 2008;77(suppl 1):13-22
Omura 
Tournigand et al. [21] reported the results of an RCT comparing FOLFIRI followed by FOLFOX6 ( $\mathrm{arm} \mathrm{A}$ ) and the reverse sequence, FOLFOX6 followed by FOFIRI (arm B) in the treatment of advanced colorectal cancer. A total of 226 patients were randomly assigned with 113 in each arm. In the first-line therapy, 3 complete responses were observed with FOLFIRI (2.8\%) and 5 with FOLFOX6 (4.5\%). The RRs were 56\% with FOLFIRI and 54\% with FOLFOX6 (not significant). The median response time was 11 months for arm A and 10.6 months for arm $B$. In the second-line therapy, the RRs were $15 \%$ with FOLFOX6 (arm A) and 4\% with FOLFIRI (arm B). The arm A patients received a median of 8 cycles $(2-23$ months) of FOLFOX6, and those in arm B received a median of 6 cycles (1-33 months) of FOLFIRI as the secondline therapy. There were no chemotherapy-related deaths. The median OS was 21.5 months for arm A and 20.6 months for arm B $(p=0.99)$. From these results, FOLFIRI and FOLFOX are now considered to have almost the same efficacy in the treatment of advanced or metastatic colorectal cancer when another regimen is used as the second-line therapy. It is important to use all of LV/5-FU, CPT-11 and L-OHP in the treatment of advanced or metastatic colorectal cancer to achieve adequate clinical results. FOLFIRI followed by FOLFOX or FOLFOX followed by FOLFIRI provide about 21 months of MST in advanced or metastatic colorectal cancer.

\section{Molecular Targeting in Chemotherapy against Advanced, Recurrent or Metastatic Colorectal Cancer}

VEGF is a proangiogenic growth factor, and has a crucial role in physiological and pathological angiogenesis. VEGF regulates both vascular proliferation and permeability and functions as an antiapoptotic factor for newly formed blood vessels. Angiogenesis is required for tumor growth and metastasis. Abnormal angiogenesis in cancer is characterized by excessive neovascularization. Consequently, VEGF represents an exciting target for cancer treatment [22].

Bevacizumab (Avastin ${ }^{\circledR}$ ) is an anti-VEGF monoclonal antibody [23]. Bevacizumab decreases tumor perfusion, vascular volume, microvascular density, interstitial fluid pressure and the number of viable, circulating endothelial cells [24].

Bevacizumab plus 5-FU/LV has resulted in a higher response and longer survival than 5-FU/LV alone in firstline metastatic colorectal cancer [25]. Hurwitz et al. [26] reported the results of an RCT to investigate the additional effects of bevacizumab on IFL in the treatment of metastatic colorectal cancer. A total of 813 patients with previously untreated metastatic colorectal cancer were randomly assigned to receive IFL plus bevacizumab $5 \mathrm{mg} / \mathrm{kg}$ every 2 weeks $(\mathrm{n}=402)$ and to receive IFL plus placebo $(\mathrm{n}=411)$. The MST was 20.3 months in the group given IFL plus bevacizumab, as compared with 15.6 months in the group given IFL plus placebo, corresponding to a hazard ratio for death of $0.66(\mathrm{p}<0.001)$. The median duration of PFS was also significantly prolonged in the IFL plus bevacizumab group as compared with the IFL plus placebo group ( $\mathrm{p}<0.001)$, and the RR in the IFL plus bevacizumab group (44.8\%) was significantly higher compared with that in the IFL plus placebo group $34.8 \%$, $\mathrm{p}=0.004)$. Bevacizumab with FOLFOX4 or capecitabine/ L-OHP (XELOX) has also been reported to prolong PFS significantly compared with a placebo and chemotherapy (10.4 vs. 7.9, $\mathrm{p}=0.0023$ ) [27]. In subgroup analysis, a significant additional effect of bevacizumab has been observed with XELOX but not with FOLFOX4. However, another study showed that bevacizumab had a synergistic effect with FOLFOX [28].

Bevacizumab is now approved in the first- or secondline chemotherapy against advanced or metastatic colorectal cancer with LV/5-FU, IFL, FOLFOX4 or XELOX [25-28]. However, the adverse effects of bevacizumab, including deep vein thrombosis and perforation of gastrointestinal tract, cannot be disregarded [29]. As the clinical effects provided by bevacizumab are not so large, further examination is needed regarding the merits and demerits of this expensive drug.

VEGF is a protein tyrosine kinase expressed on many types of tumor cell, including colorectal, breast and ovarian cancer cells [30]. There seems to be an association between upregulation of EGFR and poor clinical outcome and prognosis for human cancers. Cetuximab (Erbitux $\left.^{\circledR}\right)$ is a chimeric antibody consisting of a highly specific murine monoclonal antibody component that binds specifically to human EGFR with an affinity equal to its ligand and human IgG1 [30]. Cetuximab competes with the ligand for binding and blocks the activation of the EGFR tyrosine kinase.

Cetuximab showed disease control effects in tumors that had been refractory to CPT-11, L-OHP and 5-FU when used with CPT-11 [31]. In most of the clinical trials with cetuximab, a correlation between response and its main toxicity, an acne-like skin reaction, has been observed. 
Table 1. Summarized advances in the first-line chemotherapy against advanced or metastatic colorectal cancer

\begin{tabular}{llccc}
\hline Authors & Regimens & Patients & RR, \% & OS, months \\
\hline Scheithauer et al. [1], 1993 & BSC & 12 & 5 \\
& LV/5-FU/cisplatin & 24 & - & 11 \\
Thirion et al. [4], 2004 & 5-FU & meta-analysis & 11 & 10.5 \\
& LV/5-FU & 21 RCTs & 21 & 11.7 \\
Saltz et al. [12], 2000 & LV/5-FU & 226 & 21 & 12.6 \\
& LV/5-FU/CPT-11 (IFL) & 231 & 39 & 14.8 \\
& CPT-11 & 226 & 18 & 12.0 \\
De Gramont et al. [18], 2000 & LV5FU2 & 210 & 22.3 & 14.7 \\
& FOLFOX4 & 210 & 50.7 & 16.2 \\
Goldberg et al. [9], 2004 & IROX & 264 & 35 & 17.4 \\
& IFL & 264 & 31 & 15.0 \\
Tournigand et al. [21], 2004 & FOLFOX4 & FOLFIRI $\rightarrow$ FOLFOX6 & 113 & 19.5 \\
& FOLFOX6 $\rightarrow$ FOLFIRI & 113 & 56 & 21.5 \\
Hurwitz et al. [26], 2004 & IFL + Ava & 402 & 54 & 20.6 \\
Saltz et al. [27], 2007 & IFL + Plac & 411 & 44.8 & 20.3 \\
& FOLFOX4 or Cape + Ava & 699 & 34.8 & 15.6 \\
Van Cutsem et al. [32], 2007 & FOLFOX4 or Cape + Plac & 701 & 38 & 10.4 (PFS) \\
& FOLFIRI + Cet & 608 & 38 & 7.9 (PFS) \\
Bokemeyer et al. [33], 2007 & FOLFIRI + Plac & 609 & 46.9 & 8.9 (PFS) \\
& FOLFOX + Cet & 169 & 38.7 & 8.0 (PFS) \\
& FOLFOX + Plac & 168 & 35.6 & NR \\
& & & 35.7 & NR
\end{tabular}

Ava = Bevacizumab; Cet = cetuximab; Cape = capecitabine; Plac = placebo; NR = not reported. Thirion et al.: analyzed RCTs were reported between 1987 and 2003.

Recently, the combination effect of cetuximab as the first-line therapy against metastatic colorectal cancer with FOLFIRI or FOLFOX has been proven. Van Cutsem et al. [32] reported the results of the CRYSTAL trial in ASCO 2007. In the CRYSTAL trial, 1,217 EGFR-expressing metastatic colorectal patients were randomized to receive either cetuximab $\left(400 \mathrm{mg} / \mathrm{m}^{2}\right.$ initial dose, then 250 $\mathrm{mg} / \mathrm{m}^{2} /$ week) plus FOLFIRI (group $\mathrm{A}, \mathrm{n}=608$ ) or FOLFIRI alone (group $\mathrm{B}, \mathrm{n}=609$ ). The median PFS was significantly longer for group A compared to group B (8.9 vs. 8.0 months, $\mathrm{p}=0.036$ ). The $\mathrm{RR}$ was also significantly increased by cetuximab (46.9 vs. $38.7 \%, \mathrm{p}=0.005)$. Cetuximab in combination with FOLFIRI significantly increases the RR and significantly prolongs PFS in the firstline treatment of metastatic colorectal cancer.

Bokemeyer et al. [33] reported the results of the OPUS study. In the OPUS study, 337 patients with previously untreated EGFR-expressing metastatic colorectal cancer were enrolled. They were randomized to receive FOLFOX4 plus cetuximab $\left(400 \mathrm{mg} / \mathrm{m}^{2}\right.$ dose, then $250 \mathrm{mg} / \mathrm{m}^{2}$ every 2 weeks, group A, $n=169$ ) or FOLFOX4 alone (group $\mathrm{B}, \mathrm{n}=168$ ). The overall $\mathrm{RR}$ was $45.6 \%$ in group $\mathrm{A}$ and $35.7 \%$ in group $\mathrm{B}$. The disease control rate was $85.2 \%$ in group $\mathrm{A}$ and $81.0 \%$ in group B. The RR for the patients with grade 3 skin rash $(n=24)$ was $66.7 \%$. On the other hand, the RR for the patients with grade 0 skin rash ( $\mathrm{n}=$ 23 ) was $13 \%$. The addition of cetuximab increased the RR of FOLFOX4 in the first-line treatment of metastatic colorectal cancer. The severity of the skin rash also correlated to the clinical effects in this combination. Cetuximab is likely to be effective in the first-line chemotherapy against metastatic colorectal cancer with FOLFIRI or FOLFOX (table 1).

Saltz et al. [34] reported the effect of cetuximab (with or without CPT-11) as second-line therapy in patients who failed CPT-11 in combination with bevacizumab (BOND-2 study). The BOND-2 study was a randomized phase II trial in patients with CPT-11-refractory colorectal cancer. All patients were naïve to both bevacizumab and cetuximab. The patients in arm A received CPT-11 at the same dose and schedule as last received before study entry, plus cetuximab at a $400 \mathrm{mg} / \mathrm{m}^{2}$ loading dose, then 
weekly cetuximab $250 \mathrm{mg} / \mathrm{m}^{2}$, plus bevacizumab $5 \mathrm{mg} /$ $\mathrm{kg}$ administered every other week (CBI). The patients in arm $B$ received the same cetuximab and bevacizumab as those in arm A but without CPT-11 (CB). Forty-three patients received CBI, and 40 patients received CB. For the CBI arm, the TTP was 7.3 months and the RR 37\%; for the CB arm, the TTP was 4.9 months and the RR was $20 \%$. The OS for the CBI arm was 14.5 months and the OS for the CB-alone arm 11.4 months. The CBI showed this feasible for second-line therapy against colorectal cancer, and its activity appeared favorable.

\section{Medical Economics in the Chemotherapy against Colorectal Cancer}

Monoclonal antibody agents, including bevacizumab and cetuximab, are very expensive. Consequently, even if they demonstrate significant activity in advanced or metastatic colorectal cancer, it is necessary to estimate the cost-effectiveness of adding these agents to chemotherapy for colorectal cancer.

Tappenden et al. [35] estimated the cost-effectiveness of additive bevacizumab and/or cetuximab in the chemotherapy for untreated metastatic colorectal cancer with a systematic review. This independent health economic assessment suggests that the cost-effectiveness of bevacizumab plus IFL is unlikely to be better than GBP 46,853 per life-year gained and the cost-utility of bevacizumab plus IFL is unlikely to be better than GBP 62,857 per quality-adjusted life-year (QALY) gained. The cost-utility of bevacizumab plus LV/5-FU versus LV/5-FU is also unlikely to be better than GBP 88,658 per QALY gained.
There is no direct evidence to demonstrate whether cetuximab in combination with CPT-11 improves healthrelated quality of life in comparison to BSC or FOLFOX. Consequently, it is difficult to confirm whether cetuximab represents value for money. However, indirect comparisons suggest that the incremental cost-utility of cetuximab plus CPT-11 is unlikely to be better than GBP 30,000 per QALY gained. For the very high acquisition costs in relation to clinical benefits, the antibody agents bevacizumab and cetuximab are unlikely to represent cost-effectiveness or cost-utility.

\section{Future Perspectives in the Chemotherapy against Colorectal Cancer}

Combination chemotherapy regimens, including FOLFIRI and FOLFOX, have prolonged the survival of advanced or metastatic colorectal cancer patients 3- to 4fold compared with BSC alone. Additional monoclonal antibody agents provide little additive utility at high cost. New agents, including new macromolecule agents, smallmolecule agents and vaccines, will be introduced in the chemotherapy against colorectal cancer. Subsequently, clinical researchers will have to consider the cost-utility of these agents using QALY.

\section{Disclosure Statement}

The author declares that no financial or other conflict of interest exists in relation to the content of the article.

\section{References}

1 Scheithauer W, Rosen H, Kornek GV, et al: Randomised comparison of combination chemotherapy plus supportive care with supportive care alone in patients with metastatic colorectal cancer. BMJ 1993;306:752755.

2 Kubota T, Fujita S, Kodaira S, et al: Antitumor activity of fluoropyrimidines and thymidylate synthetase inhibition. Jpn J Cancer Res 1991;82:476-482.

3 Spears CP, Gustavsson BG, Berne M: Mechanisms of innate resistance to thymidylate synthase inhibition after 5-fluorouracil. Cancer Res 1988;48:5894-5900.
4 Thirion P, Michiels S, Pignon JP, et al: Modulation of fluorouracil by leucovorin in patients with advanced colorectal cancer: an updated meta-analysis. J Clin Oncol 2004; 22:3766-3775.

5 Madajewicz S, Petrelli N, Rustum YM, et al: Phase I-II trial of high-dose calcium leucovorin and 5-fluorouracil in advanced colorectal cancer. Cancer Res 1984;44:4667-4669.

6 O'Connell MJ: A controlled clinical trial including folinic acid at two distinct dose levels in combination with 5 -fluorouracil (5FU) for the treatment of advanced colorectal cancer: experience of the Mayo Clinic and North Central Cancer Treatment Group. Adv Exp Med Biol 1988;244:173-184.
De Gramont A, Krulik M, Cady J, et al: Highdose folinic acid and 5-fluorouracil bolus and continuous infusion in advanced colorectal cancer. Eur J Cancer Clin Oncol 1988;24:1499-1503.

8 Weh HJ, Wilke HJ, Dierlamm J, et al: Weekly therapy with folinic acid (FA) and highdose 5-fluorouracil (5-FU) 24-hour infusion in pretreated patients with metastatic colorectal cancer: a multicenter study by the Association of Medical Oncology of the German Cancer Society (AIO). Ann Oncol 1994; 5:233-237. 
-9 Goldberg RM, Sargent DJ, Morton RF, et al: A randomized controlled trial of fluorouracil plus leucovorin, irinotecan, and oxaliplatin combinations in patients with previously untreated metastatic colorectal cancer. J Clin Oncol 2004;22:23-30.

10 Kunimoto T, Nitta K, Tanaka T, et al: Antitumor activity of 7-ethyl-10-[4-(1-piperidino)-1-piperidino] carbonyloxy-camptothecin, a novel water-soluble derivative of camptothecin, against murine tumors. Cancer Res 1987;47:5944-5927.

-11 Wang JC: DNA topoisomerases. Annu Rev Biochem 1985;54:665-697.

12 Saltz LB, Cox JV, Blanke C, et al; Irinotecan Study Group: Irinotecan plus fluorouracil and leucovorin for metastatic colorectal cancer. N Engl J Med 2000;343:905-914.

-13 André T, Louvet C, Maindrault-Goebel F, et al; GERCOR: CPT-11 (irinotecan) addition to bimonthly, high-dose leucovorin and bolus and continuous-infusion 5-fluorouracil (FOLFIRI) for pretreated metastatic colorectal cancer. Eur J Cancer 1999;35:1343-1347.

14 Kidani Y, Inagaki K, Iigo M, et al: Antitumor activity of 1,2-diaminocyclohexane-platinum complexes against sarcoma-180 ascites form. J Med Chem 1978;21:1315-1318.

$\checkmark 15$ Habala L, Galanski M, Yasemi A, et al: Synthesis and structure-activity relationships of mono- and dialkyl-substituted oxaliplatin derivatives. Eur J Med Chem 2005;40:11491155.

16 De Gramont A, Tournigand C, Louvet C, et al; GERCOD: Oxaliplatin, folinic acid and 5fluorouracil (folfox) in pretreated patients with metastatic advanced cancer. Rev Med Interne 1997;18:769-775.

$\checkmark 17$ André T, Bensmaine MA, Louvet C, et al: Multicenter phase II study of bimonthly high-dose leucovorin, fluorouracil infusion, and oxaliplatin for metastatic colorectal cancer resistant to the same leucovorin and fluorouracil regimen. J Clin Oncol 1999;17: 3560-3568.

$\checkmark 18$ De Gramont A, Figer A, Seymour M, et al: Leucovorin and fluorouracil with or without oxaliplatin as first-line treatment in advanced colorectal cancer. J Clin Oncol 2000; 18:2938-2947.
19 Tournigand C, Cervantes A, Figer A, et al: OPTIMOX1: a randomized study of FOLFOX4 or FOLFOX7 with oxaliplatin in a stop-and-go fashion in advanced colorectal cancer - a GERCOR study. J Clin Oncol 2006;24:394-400.

20 Maindrault-Goebel F, Liedo G, Chibaudel B, et al: Final results of OPTIMOX2: a large randomized phase II study of maintenance therapy or chemotherapy-free interval (CFI) after FOLFOX in patients with metastatic colorectal cancer (MCRC) - a GERCOR study. ASCO 2007, abstract 4013.

21 Tournigand C, André T, Achille E, et al: FOLFIRI followed by FOLFOX6 or the reverse sequence in advanced colorectal cancer: a randomized GERCOR study. J Clin Oncol 2004;22:229-237.

22 Ferrara N, Gerber HP, LeCouter J: The biology of VEGF and its receptors. Nat Med 2003;9:669-976.

23 Presta LG, Chen H, O’Connor SJ, et al: Humanization of an anti-vascular endothelial growth factor monoclonal antibody for the therapy of solid tumors and other disorders. Cancer Res 1997;57:4593-4599.

24 Willett CG, Boucher Y, di Tomaso E, et al: Direct evidence that the VEGF-specific antibody bevacizumab has antivascular effects in human rectal cancer. Nat Med 2004;10: 145-147.

25 Kabbinavar FF, Ellis LM: Can inhibition of angiogenic pathways increase the efficacy of intravenous 5-fluorouracil-based regimens? Clin Colorectal Cancer 2004;4(suppl 2):S69S73.

26 Hurwitz H, Fehrenbacher L, Novotny W, et al: Bevacizumab plus irinotecan, fluorouracil, and leucovorin for metastatic colorectal cancer. N Engl J Med 2004;350:2335-2342.

27 Saltz LB, Clarke S, Diaz-Rubio, et al: Bevacizumab (Bev) in combination with XELOX or FOLFOX4: update efficacy results from XELOX-1/NO16966, a randomized phase III trial in first-line metastatic colorectal cancer. ASCO 2007, abstract 4028 .
28 Giantonio BJ, Catalano PJ, Meropol NJ, et al: Bevacizumab in combination with oxaliplatin, fluorouracil, and leucovorin (FOLFOX4) for previously treated metastatic colorectal cancer: results from the Eastern Cooperative Oncology Group Study E3200. J Clin Oncol 2007;25:1539-1544.

29 Gordon MS, Cunningham D: Managing patients treated with bevacizumab combination therapy. Oncology 2005;69(suppl 3):2533.

30 Goldstein NI, Prewett M, Zuklys K, et al: Biological efficacy of a chimeric antibody to the epidermal growth factor receptor in a human tumor xenograft model. Clin Cancer Res 1995;1:1311-1318.

31 Pfeiffer P, Nielsen D, Yilmaz M, et al: Cetuximab and irinotecan as third line therapy in patients with advanced colorectal cancer after failure of irinotecan, oxaliplatin and 5-fluorouracil. Acta Oncol 2007;46: 697-701.

32 Van Cutsem E, Nowacki M, Lang I, et al: Randomized phase III study of irinotecan and 5-FU/FA with or without cetuximab in the first-line treatment of patients with metastatic colorectal cancer (mCRC): the CRYSTAL trial. ASCO 2007, abstract 4000.

33 Bokemeyer C, Bondarenko I, Makhson A, et al: Cetuximab plus 5-FU/FA/oxaliplatin (FOLFOX-4) versus FOLFOX-4 in the firstline treatment of metastatic colorectal cancer (mCRC): OPUS, a randomized phase II study. ASCO 2007, abstract 4035.

34 Saltz LB, Lenz HJ, Kindler HL, et al: Randomized phase II trial of cetuximab, bevacizumab, and irinotecan compared with cetuximab and bevacizumab alone in irinotecan-refractory colorectal cancer: the BOND-2 study. J Clin Oncol 2007;25:45574561.

35 Tappenden P, Jones R, Paisley S, et al: Systematic review and economic evaluation of bevacizumab and cetuximab for the treatment of metastatic colorectal cancer. Health Technol Assess 2007;11:1-128, iii-iv. 